\title{
PEIXES DO ESTUÁRIO DO RIO PARIPE (ITAMARACÁ-PE)
}

\author{
ANTÔNIO LEMOS DE VASCONCELOS FILHO \\ FERNANDO DUARTE ACIOLI \\ Departamento de Oceanografia da UFPE \\ DINALVA DE SOUZA GUEDES \\ Departamento de Pesca da UFRPE
}

\section{RESUMO}

O presente estudo faz um reconhecimento de várias espécies de peixes que ocorrem no estuário do Rio Paripe, localizado no extremo Sul do Canal de Santa Cruz na área de Vila Velha em Itamaracá-PE. O material consta do acervo da coleção ictiológica do Departamento de Oceanografia da UFPE, tendo sido os mesmos coletados na desembocadura do rio por meio de uma rede de arrasto com $14 \mathrm{~m}$ de comprimento e malha de $15 \mathrm{~mm}$. Foram determinados e revisados 146 exemplares sendo agrupados em 19 famílias, 24 gêneros e 29 espécies de peixes. Os resultados obtidos permitiram a apresentação de uma lista de espécies, incluindo para cada uma, o nome científico, nome comum regional, comprimento padrão, distribuição geográfica e o número de exemplares examinados. Observouse a presença de indivíduos de pequeno e médio porte. As famílias de peixes abundantes em número de indivíduos e espécies foram: Engraulidae, Centropomidae, Bothidae e Tetraodontidae.

Palavras chave: Peire, taxonomia, estuário, tropical

\section{ABSTRACT}

Fishes of the Estuary of the River Paripe (Itamaracá - PE)

The present study makes a recognition of severals fishes that occur in the Paripe River stuary located in the extreme south of Santa Cruz channel in Vila Velha area at Itamaracá-PE. The material consist of a large amount of the ictiological collection belong the Department of the Oceanography of the Federal University of Pernambuco. The material had been collected in the river mouth through a haul net with $14 \mathrm{~m}$, lenght and $15 \mathrm{~mm}$ mesh. 146 specimens were identified and revised being grouped in 19 families, 24 genera and 29 species of fishes. The results obtained allowed the presentation of a species list, including for each one, the scientific name, regional name, standard length, geographical fistribution and the number of the species examined. The presence of the small and medium specimens was observed. The fishes families abundant in specimens and species number were: Engraulidae, Centropomidae, Bothidae e Tetraodontidae.

In words: Fish, taxonomy, estuary, tropical 


\section{INTRODUÇÃO}

No Nordeste do Brasil, algumas pesquisas relacionadas com a Taxonomia e Ecologia de peixes são reportadas, por Eskinazi $(1967 / 69)$ e Oliveira (1979); com relação a área de Itamaracá-PE, destacam Eskinazi (1972) e Azevedo e Guedes (1980). Quanto ao Rio Paripe, o qual desemboca no Canal de Santa Cruz, poucos estudos são evidenciados, como é o caso de Santos (1988), que apresenta uma relação da fauna bêntica do estuário do Rio Paripe, demostrando que os Moluscos e Artropodos são os mais abundantes; Coelho e Santos (1990), determinaram os Crustáceos Decápodos e Estomatópodos desse ambiente; Fernandes (1990), apresenta uma lista preliminar dos Moluscos Gastropódos da mesma área; Silva (1992), relata em sua pesquisa, a variação sazonal da produção, biomassa e densidade do fitoplâncton e Lacerda (1994), determinou a variação diurna da população fitoplanctônica e sua relação com as condições hidrológicas desse ambiente.

Com o objetivo de contribuir para o enriquecimento dos estudos sobre a fauna ictiológica da área de Itamaracá, foi feito uma determinação e revisão dos peixes do estuário do Rio Paripe, provenientes da coleção ictiológica do Departamento de Oceanografia da UFPE, uma vez que os trabalhos sobre a composição ictiológica estão direcionados notadamente, para o Canal de Santa Cruz, podendo ainda estes estudos, serem ampliados com novas coletas de peixes.

\section{CARACTERIZAÇÃO DA ÁREA}

O Rio Paripe (Fig.1), é um dos poucos rios que nascem na liha de Itamaracá, localizado $7^{\circ} 48^{\prime} 38^{\prime \prime}$ latitude Sul e $34^{\circ} 51^{\prime} 27^{\prime \prime}$ longitude Oeste, desaguando nas proximidades Sul do Canal de Santa Cruz. Possui $4 \mathrm{Km}$ de extensão e seu estuário apresenta $1,6 \mathrm{Km}$ de comprimento e $0,55 \mathrm{Km}$ na sua parte mais larga (Coelho e Santos, 1990).

Segundo Macêdo et alii (1973), a temperatura média está em torno de $29^{\circ} \mathrm{C}$ na baixa-mar e $28,8^{\circ} \mathrm{C}$ na preamar; com relação a salinidade observase variação entre $12,18 \%$ a $32,33 \%$. O oxigênio dissolvido esteve entre os índices 3,58 e 4,97 ml/h, observando-se que a influência marinha é marcante.

A vegetação predominante é o manguezal, formada em sua maior parte pela Rhizophora mangle Linnaeus (mangue-vermelho), Laguncularia racemosa Gaerth (mangue-branco) e Avicennia shaueriana Stapf e Leechaman (manguesiriuba), sendo a primeira dominante sobre as demais (Silva, 1992).

\section{MATERIAL E MÉTODOS.}

$\mathrm{O}$ aporte ictiológico em estudo neste trabalho, consta de 146 exemplares pertencentes à coleção Ictiológica da Seção de Nécton do Departamento de Oceanografia da Universidade Federal de Pernambuco. O material é proveniente do estuário do Rio Paripe, localizado no extremo Sul da Ilha de Itamaracá-PE. 


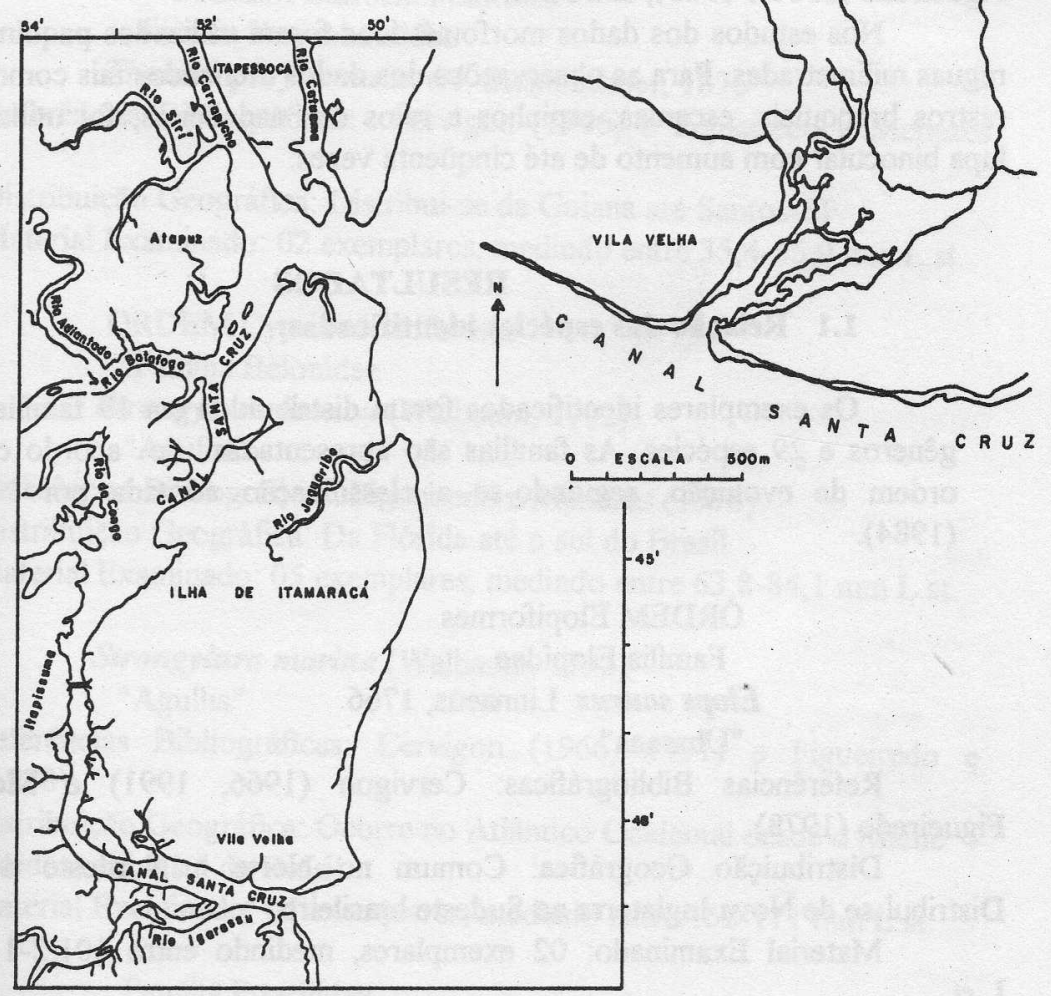

Figuro-1 - MAPA DA AREA ESTUDADA SEGUNDO COELHO \& SANTOS, 1990 
A captura dos exemplares foi feita, geralmente, por meio de uma rede de arrasto com $14 \mathrm{~m}$ de comprimento e malha de $15 \mathrm{~mm}$, na área da desembocadura do rio, principalmente nos anos de 1969, 1976 e 1994. Após as coletas os indivíduos capturados foram inseridos em recipientes de vidro, fixados em formol a $4 \%$ e devidamente rotulados; no laboratório os peixes foram lavados em água corrente e preservados em álcool etílico a $75 \%$, para posteriores análises taxonômicas.

$\mathrm{Na}$ identificação das espécies de peixes foram consultados os trabalhos especializados em taxonomia: Cervigon (1966, 1991 e 1993), Figueiredo e Menezes (1978 e 1980), Hoese (1978), Randal e Vergara (1978) e Menezes e Figueiredo (1980 e 1985), entre outros.

Nos estudos dos dados morfométricos foram utilizados paquímetros ou réguas milimetradas. Para as observações dos dados merísticos tais como: dentes, rastros branquiais, escamas, espinhos e raios das nadadeiras, foi utilizada uma lupa binocular com aumento de até cinqüenta vezes.

\section{RESULTADOS \\ 1.1 Relação das espécies identificadas:}

Os exemplares identificados foram distribuídos em 19 familias, 24 gêneros e 29 espécies. As famílias são apresentadas de acordo com a ordem de evolução, seguindo-se a classificação adotada por Nelson (1984).

ORDEM Elopiformes

Familia Elopidae

Elops saurus Linnaeus, 1766

"Ubarana"

Referências Bibliográficas: Cervigon (1966, 1991) e Menezes e Figueiredo (1978).

Distribuição Geográfica: Comum no Norte e Nordeste do Brasil. Distribui-se de Nova Inglaterra ao Sudeste brasileiro.

Material Examinado: 02 exemplares, medindo entre 101,2-115,3 mm L.st.

ORDEM Clupeiformes

Família Clupeidae

Harengula clupeola Cuvier, 1829

"Sardinha-cascuda"

Referências Bibliográficas: Cervigon $(1966,1991)$ e Figueiredo e Menezes (1978).

Distribuição Geográfica: Ocorre da Flórida ao Estado de São Paulo.

Material Examinado: 01 exemplar medindo $68,4 \mathrm{~mm}$ L.st. 


\section{Família Engraulidae}

Cetengraulis edentulus (Cuvier, 1829)

"Manjuba, arenque"

Referências Bibliográficas: Cervigon $(1966,1991)$ e Menezes e Figueiredo (1978). Catarina.

Distribuição Geográfica: Caribe e do Panamá á costa do Estado Santa

Material Examinado: 20 exemplares, medindo entre 59,3-94,2 mm L.st.

ORDEM Batrachoidiformies

Família Batrachoididae

Thalassophryne nattereri Steindachner, 1876

(1978).

Referências Bibliográficas: Cervigon (1966) e Figueiredo e Menezes

Distribuição Geográfica: Distribui-se da Guiana até Santos-SP

Material Examinado: 02 exemplares, medindo entre 35,4-55,9 $\mathrm{mm}$ L.st.

ORDEM Cyprinodontiformes

Família Belonidae

Strongylura timucu (Walbaum, 1792)

"Agulha"

Referências Bibliográficas: Figueiredo e Menezes (1978).

Distribuição Geográfica: Da Flórida até o sul do Brasil.

Material Examinado: 05 exemplares, medindo entre 63,8-84,1 mm L.st.

Strongylura marina (Walbaum, 1792)

"Agulha"

Referências Bibliográficas: Cervigon (1966, 1991) e Figueiredo e enezes (1978).

Distribuição Geográfica: Ocorre no Atlântico Ocidental desde o Maine Etrados Unidos) até o sul do Brasil.

Material Examinado: 04 exemplares, medindo entre 152-171 mm L.st.

Familia Poecilidae

Poecilia vivipara Bloch e Schneider, 1801

"Guaru"

Referências Bibliográficas: Cervigon (1966, 1991).

Distribuição Geográfica: Aruba, Curaçao, Bonaire e as Ilhas e costa da enecuela até o Rio do Prata, Argentina.

Material Examinado: 04 exemplares, medindo entre 36,1- 53,3 mm L.st.

ORDEM Atheriniformes

Família Atherinidae 
Xenomelaniris brasiliensis (Quoy e Gaimard, 1824)

"Peixe-rei"

Referências Bibliográficas: Cervigon (1966, 1991) e Figueiredo e Menezes ( 1978).

Distribuição Geográfica: Ocorre na Venezuela até o Rio Grande do Sul.

Material Examinado: 02 exemplares, medindo entre 27,1- 45,3 mm L.st.

ORDEM Perciformes

Família Centropomidae

Centropomus undecimalis (Bloch, 1792)

"Camorim cabo-de-machado"

Referências Bibliográficas: Cervigon (1966, 1991) e Figueiredo e Menezes (1980).

Distribuição Geográfica: Distribui-se da Flórida (sul) até ao sul do Brasil.

Material Examinado: 11 exemplares, medindo entre 47,7-126,3 mm L.st.

Centropomus parallelus Poey, 1860

"Camorim-corcunda"

Referências Bibliográficas: Cervigon $(1966,1991)$ e Figueiredo e Menezes (1980).

Distribuição Geográfica: Distribui-se da Flórida até o sul do Brasil.

Material Examinado: 02 exemplares, medindo entre 119,9-124,3 mm

L.st.

Família Carangidae

Selene vomer (Linnaeus, 1758)

"Peixe galo-de-penacho" (1980).

Referências Biblográficas: Cervigon (1966) e Figueiredo e Menezes

Distribuição Geográfica: Ocorre do Maine ao Uruguai.

Material Examinado: 01 exemplar, medindo 36,3 mm L.st

Família Lutjanidae

Lutjanus jocu (Bloch e Schneider, 1801)

"Vermelho"

Referências Bibliográficas: Menezes e Figueiredo (1980).

Distribuição Geográfica: Da Nova Inglaterra ao sudeste do Brasil.

Material Examinado: 03 exemplares, medindo entre 38-48,1 $\mathrm{mm}$ L.st.

Lutjanus apodus (Walbaum, 1792)

Referências Bibliográficas: Cervigon $(1966,1993)$.

Distribuição Geográfica: Nos dois lados do Atlântico. Desde as Bermudas e Massachusetts até a Bahia (Brasil). 
Material Examinado: 05 exemplares, medindo entre 53,7-73,1 mm L.st.

\section{Lutjanus griseus (Linnaeus, 1758) \\ "Caranha"}

Referências Bibliográficas: Cervigon $(1966,1993)$ e Menezes e Figueiredo (1980).

Distribuição Geográfica: Distribui-se no Atlântico Ocidental e foi assinalado da Nova Inglaterra ao Sudeste do Brasil.

Material Examinado: 05 exemplares, medindo entre 34,6-54,7 mm L.st.

Família Gerreidae

Ulaema lefroyi (Goode, 1874)

"Carapicu"

Referências Bibliográficas: Cervigon $(1966,1993)$ e Menezes e Figueiredo (1980).

Distribuição Geográfica: Bahamas ao sudeste do Brasil.

Material Examinado: 05 exemplares, medindo entre 32,7-64 mm L.st.

Eugerres brasilianus (Valenciennes, 1830)

"Carapeba-prateada, Caratinga"

Referências Bibliográficas: Cervigon (1966, 1993) e Menezes e Figueiredo (1980).

Distribuição Geográfica: Distribui-se das Antilhas ao Sul do Brasil.

Material Examinado: 01 exemplar medindo 74,6 mm L.st.

Eucinostomus gula (Cuvier, 1830)

"Carapicu"

Referências Bibliográficas: Cervigon $(1966,1993)$ e Menezes e Figueiredo (1980).

Distribuição Geográfica: Ocorre de Nova Inglaterra até a Argentina.

Material Examinado: 03 exemplares, medindo entre 56,7-80,4 mm L.st.

Eucinostomus argenteus (Baird e Girard, 1854)

"Carapicu "

Referências Bibliográficas: Cervigon (1966, 1993) e Menezes e Eyeiredo (1980).

Distribuição Geográfica: Encontrado no Pacífico leste e no Atlântico eidental,estendendo-se de Nova Jersey ao sul do Brasil.

Material Examinado: 01 exemplar, medindo 104,5 mm L.st.

Familia Ephippididae

Chaetodipterus faber (Broussonet, 1782)

"Paru, Enxada " 
Referências Bibliográficas: Cervigon (1966, 1993) e Menezes e Figueiredo (1985).

Distribuição Geográfica: No Atlântico Ocidental, distribui-se da Nova Inglaterra ao sul do Brasil.

Material Examinado: 03 exemplares, medindo entre 25-39,5 mm L.st.

Família Mugilidae

Mugil curema Valenciennes, 1836

"Tainha, Parati"

Referências Bibliográficas: Cervigon (1966) e Menezes e Figueiredo (1985).

Distribuição Geográfica: Em ambos os lados do Atlântico e no Pacífico Leste. No Atlântico Ocidental estende-se da Nova Inglaterra ao Sul do Brasil.

Material Examinado: 02 exemplares, medindo entre 80,1-81,2 mm L.st.

Família Sphyraenidae

Sphyraena barracuda (Walbaum, 1792)

"Barracuda"

Referências Bibliográficas: Cervigon $(1966,1993)$ e Menezes e Figueiredo (1985).

Distribuição Geográfica: Distribui-se no Atlântico Ocidental estendendose de Massachusetts até o sul do Brasil.

Material Examinado: 01 exemplar medindo 47,1 mm L.st.

Família Eleotrididae

Eleotris pisonis (Gmelin, 1789)

Referências Bibliográficas: Cervigon (1966) e Menezes e Figueiredo (1985).

Distribuição Geográfica: Distribui-se nas Bahamas, Bermudas e desde a Carolina do Sul até Ubatuba-SP. Conhecida na região do baixo Amazonas.

Material Examinado: 02 exemplares, medindo entre 56,8-74,3 $\mathrm{mm} \mathrm{L.st.}$

Erotelis smaragdus (Valenciennes, 1837)

Referências Bibliográficas: Menezes e Figueiredo (1985), Böhlke e Chaplin (1968), Hoese (1978).

Distribuição Geográfica: desde as Bahamas e Flórida até o Brasil.

Material Examinado: 03 exemplares, medindo entre 45,1-60,3 mm L.st.

\section{Familia Gobiidae}

Gobionellus smaragdus (Valenciennes, 1837)

Referências Bibliográficas: Cervigon (1966) e Menezes e Figueiredo (1985).

Distribuição Geográfica: Da Carolina do Sul até Cananéia-SP.

Material Examinado: 06 exemplares, medindo entre 55,7-56,6 mm L.st. 


\title{
Bathygobius soporator (Valenciennes, 1837)
}

\author{
"Emboré, Mororó"
} (1985).

Referências Bibliográficas: Cervigon (1966) e Menezes e Figueiredo

Distribuição Geográfica: Ocorre nos dois lados do Atlântico, Pacífico tropical americano, na costa leste da América, da Flórida ao Rio Grande do Sul.

Material Examinado: 09 exemplares, medindo entre 45,1-101,5 mm L.st.

\section{ORDEM Pleuronectiformes}

Família Bothidae

Syacium micrurum Ranzani, 1842

"Linguado"

Referências Bibliográficas: Cervigon (1966) e Randal e Vergara (1978).

Distribuição Geográfica: Golfo do México e Venezuela.

Material Examinado: 13 exemplares, medindo entre 54,3- 129,2 mm

L.st.

\section{Citharichthys spilopterus Gunther, 1862}

"Sôlha"

Referências Bibliográficas: Cervigon (1966).

Distribuição Geográfica: desde Nova Jersey até Santos no Brasil, e todo Golfo do México.

Material Examinado: 06 exemplares, medindo entre 70,6-93 mm L.st.

\section{Familia Soleidae}

Achirus lineatus (Linnaeus, 1758)

"Solha"

Referências Bibliográficas: Cervigon (1966).

Distribuição Geográfica: desde a Flórida até o Uruguai e por todo o Golfo do México.

Material Examinado: 05 exemplares, medindo entre 40,6-55,6 mm L.st.

\section{ORDEM Tetraodontiformes}

Família Tetraodontidae

Sphoeroides testudineus (Linnaeus, 1758)

"Baiacu"

Referências Bibliográficas: Cervigon (1966).

Distribuição Geográfica: Ao longo da Costa Atlântica, desde a Flórida até o Brasil.

Material Examinado: 19 exemplares, medindo entre 14,5-90 mm L.st. 
1.2 Número de exemplares e variação de comprimento- padrão dos peixes estudados.

De acordo com as medições realizadas com exemplares examinados, observa-se que a variação do comprimento-padrão esteve entre $14,5 \mathrm{~mm}$ em Sphoeroides testudineus e $171,0 \mathrm{~mm}$ em Strongylura marina, sendo o representante de maior tamanho no presente trabalho. Dentre as espécies analisadas, as que ocorreram com maior número de exemplares no Rio Paripe foram: Cetengraulis edentulus com 20 exemplares, Sphoeroides testudineus com 19 exemplares, Syacium micrurum com 13 exemplares e Centropomus undecimalis com 11 exemplares.

As espécies com apenas um exemplar coletado foram: Harengula clupeola, Selene vomer, Eugerres brasilianus, Eucinostomus argenteus e Sphyraena barracuda Tabela 1.

\section{CONCLUSÕES}

1 - Do total de 146 exemplares coletados foram identificados 19 famílias, 24 gêneros e 29 espécies.

2 - Das famílias determinadas, $43 \%$ pertencem a Ordem Perciformes.

3 - A fauna íctica da área é muito variada em espécimes de pequeno e médio porte, sendo capturados peixes com comprimento padrão mínimo de $14,5 \mathrm{~mm}$ observado em Sphoeroides testudineus, enquanto que algumas espécies como Strongylura marina, foi representada por exemplares com171 mm.

4 - Quanto ao número de exemplares destacaram-se: Cetengraulis edentulus, Sphoeroides testudineus, Syacium micrurum e Centropomus undecimalis sendo está última espécie de alto valor comercial.

5 - As espécies identificadas podem ser consideradas de ampla distribuição geográfica no Atlântico Ocidental Tropical.

\section{REFERÊNCIAS BIBLIOGRÁFICAS}

AZEVEDO-ARAÚJO, S.B. e GUEDES, D.S. Estudo ecológico da região de Itamaracá Pernambuco, PE, Brasil. X Novas Ocorrências de peixes. Trabalhos Oceanográficos da Universidade Federal de Pernambuco, Recife, 15: 331-342, 1980.

BOHLKE, J.E. e CHAPLIN, C.C.G. Fishes of the Bahamas. Wynnewood, The Academy of Natural Sciences of Philadelphia, 1968. $771 \mathrm{p}$.

CAVALCANTI, L.B.; COELHO, P.A.; ESKINAZI-LEÇA, E.; LUNA, J.A.C.; MACÊDO, S.J.; PARANAGUÁ, M.N. Condiciones ecologicas en el area de Suape (Pernambuco-Brasil). In: SEMINÁRIO SOBRE EL ESTUDIO CIENTIFICO E IMPACTO HUMANO EN EL ECOSISTEMA DE MANGLARES, Cali, 1978. Memorias. Mondivideu, UNESCO, 1980. p.243-56. 
TABELA 1. Variação do número de exemplares e do comprimento-padrão das Familias/Espécies de peixes na área estudada.

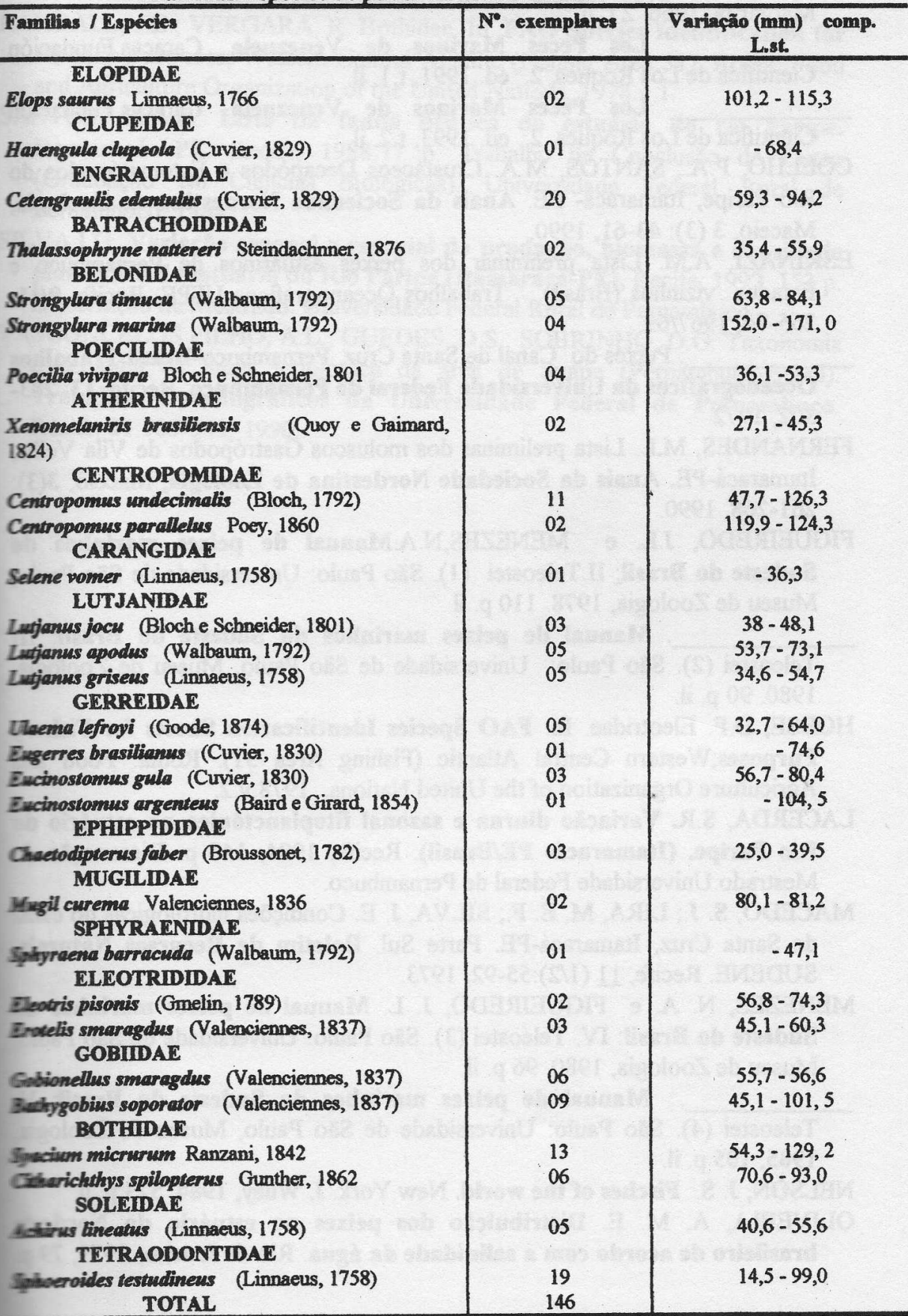


CERVIGON, F.M. Los Peces Marinos de Venezuela. Caracas.Fundación La Salle de Ciencias Naturales, Estación de Investigaciones Marinas de Margarita, 1966. 2 t. il.

Los Peces Marinos de Venezuela. Caracas.Fundación Científica de Los Roques, 2. ed. 1991, t.1. il.

Los Peces Marinos de Venezuela. Caracas.Fundación Científica de Los Roques, 2. ed. 1993, t.2. il.

COELHO, P.A.; SANTOS, M.A. Crustáceos Decapódos e Estomatópodos do Rio Paripe, Itamaracá- PE. Anais da Sociedade Nordestina de Zoologia, Maceió, 3 (3): 43-61, 1990.

ESKINAZI, A.M. Lista preliminar dos peixes estuarinos de Pernambuco e Estados vizinhos (Brasil). Trabalhos Oceanográficos UFPE. Recife, 9/11: 265-74, 1967/69.

. Peixes do Canal de Santa Cruz, Pernambuco-Brasil.Trabalhos

Oceanográficos da Universidade Federal de Pernambuco. Recife, 13: 283302, 1972.

FERNANDES, M.L. Lista preliminar dos moluscos Gastrópodos de Vila Velha, Itamaracá-PE. Anais da Sociedade Nordestina de Zoologia Maceió, 3(3): 261-268, 1990.

FIGUEIREDO, J.L. e MENEZES,N.A.Manual de peixes marinhos do Sudeste do Brasil: II.Teleostei (1). São Paulo: Universidade de São Paulo, Museu de Zoologia, 1978. 110 p. il

Manual de peixes marinhos do Sudeste do Brasil: III

Teleostei (2). São Paulo: Universidade de São Paulo, Museu de Zoologia, 1980.90 p. il.

HOESE, D.F. Eleotridae. In: FAO Species Identification Sheets for Fishery Purposes; Western Central Atlantic (Fishing Area 31). Roma: Food and Agriculture Organization of the United Nations, 1978.v.2.

LACERDA, S.R. Variação diurna e sazonal fitoplanctônica no estuário do Rio Paripe. (Itamaracá- PE/Brasil). Recife, 1994, 146 p. Dissertação de Mestrado.Universidade Federal de Pernambuco.

MACEDO, S. J.; LIRA, M. E. F.; SILVA, J. E. Condiç̃̃es hidrológicas do canal de Santa Cruz, Itamaracá-PE. Parte Sul. Boletim de Recursos Naturais. SUDENE. Recife, 11 (1/2):55-92, 1973.

MENEZES, N. A. e FIGUEIREDO, J. L. Manual de peixes marinhos do Sudeste do Brasil: IV. Teleostei (3). São Paulo: Universidade de São Paulo, Museu de Zoologia, 1980, 96 p. il.

Manual de peizes marinhos do Sudeste do Brasil: V. Teleostei (4). São Paulo: Universidade de São Paulo, Museu de Zoologia, 1985,105 p. il.

NELSON, J. S. Fisches of the world. New York. J. Wiley, 1984. 523 p. il.

OLIVEIRA, A. M. E. Distribuição dos peixes no estuário do Nordeste brasileiro de acordo com a salinidade da água. Rio de Janeiro, 1979, 79 p. 
il. Dissertação (Mestrado em Ciências Biológicas) - Museu Nacional, Universidade Federal do Rio de Janeiro, Rio de Janeiro, 1979.

RANDALL, J.E.; VERGARA, R. Bothidae. In: FAO Species Identification for Fishery Purposes; Western Central Atlantic (Fishing Area 31). Roma: Food and Agriculture Organization of the United Nations, 1978.v.1.

SANTOS, M.A.C. Lista da fauna bêntica do estuário do rio Paripe. Itamaracá- PE. Recife, 1988.77 p. Trabalho de Conclusão do Curso (Graduação em Ciências Biológicas). Universidade Federal Rural de Pernambuco, 1988.

SILVA,I.G. Variação sazonal e espacial da produção, biomassa e densidade plantônica do estuário do Rio Paripe (Itamaracá-PE), Recife, 1992. 153 p. Dissertação de Mestrado. Universidade Federal Rural de Pernambuco.

VASCONCELOS FILHO, A.L; GUEDES, D.S.; SOBRINHO, D.G. Taxonomia e ecologia da fauna ictiológica da área de Suape (Pernambuco-Brasil). Trabalhos Oceanográficos da Universidade Federal de Pernambuco. Recife, 21:305-343, 1990. 